\title{
The Effects of Interruptions on Oncologists' Patient Assessment and Medication Ordering Practices
}

\author{
Patricia L. Trbovich ${ }^{1,2,3, *}$, PhD; Melissa C. Griffin ${ }^{1}$, MHSc; Rachel E. White ${ }^{1}$, \\ MA; Venetia Bourrier ${ }^{4}$, BSc, Pharm, FCSHP; Dhali Dhaliwal ${ }^{4}$, MD; \\ Anthony C. Easty, PhD, PEng1, 2 \\ ${ }^{1}$ HumanEra, Techna, University Health Network, Toronto, Ontario, Canada \\ ${ }^{2}$ Institute of Biomaterials and Biomedical Engineering, Faculty of Medicine, \\ University of Toronto, Toronto, Ontario, Canada \\ ${ }^{3}$ Health Policy, Management and Evaluation, Faculty of Medicine, University of \\ Toronto, Toronto, Ontario, Canada \\ ${ }^{4}$ Cancer Care Manitoba, Winnipeg, Manitoba, Canada
}

Submitted December 2011. Accepted for publication October 2012.

\begin{abstract}
Interruptions are causal factors in medication errors. Although researchers have assessed the nature and frequency of interruptions during medication administration, there has been little focus on understanding their effects during medication ordering. The goal of this research was to examine the nature, frequency, and impact of interruptions on oncologists' ordering practices. Direct observations were conducted at a Canadian cancer treatment facility to (1) document the nature, frequency, and timing of interruptions during medication ordering, and (2) quantify the use of coping mechanisms by oncologists. On average, oncologists were interrupted $17 \%$ of their time, and were frequently interrupted during safety-critical stages of medication ordering. When confronted with interruptions, oncologists engaged/multitasked more often than resorting to deferring/blocking. While some interruptions are necessary forms of communication, efforts must be made to reduce unnecessary interruptions during safety-critical tasks, and to develop interventions that increase oncologists' resiliency to inevitable interruptions.
\end{abstract}

Keywords: interruptions, medication ordering, direct observation, medical oncology, medication safety

\section{INTRODUCTION}

Medication errors involving high-risk drugs such as those of chemotherapy are a significant cause of medical injuries, and are consequently a major safety concern for the health care system $[1,2]$. Interruptions have been identified as frequent contributors to medication errors and the consequent injuries $[3,4,5]$. Reports, including the

*Corresponding author: Patricia L Trbovich, University Health Network, 4th Floor, R. Fraser Elliott Building, 190 Elizabeth Street, Toronto, ON, Canada M5G 2C4. Phone: (416) 340-4800 ext7180. Fax: (416) 340-3595; E-mail: patricia.trbovich@uhn.ca. Other authors: melissa.griffin@uhn.ca; rachel.white@uhn.ca; venetia.bourrier@cancercare.mb.ca; dhali.dhaliwal@cancercare.mb.ca; tony.easty@uhn.ca. 
Institute of Medicine Report [1], the Agency for Healthcare Research and Quality's report on The Effect of Health Care Working Conditions on Patient Safety [6], and United States Pharmacopeia MEDMARX reports [7, 8], identify interruptions and distractions as factors contributing to medical errors. Distractions are included as causative factors in $47 \%$ of medication errors recorded in MEDMARX, and are the most frequently reported factors contributing to patient harm [7]. Thus, interruptions are a well-established patient safety issue.

Although researchers have assessed the nature and frequency of interruptions encountered by nurses throughout stages of the medication process, there has been little focus on understanding the effects of interruptions on physicians' medication ordering practices. Understanding the impact of interruptions on medication ordering is important given that prescribing errors have been identified as the most common form of error committed, representing nearly one third of medication errors [9]. Oncology is a particularly high-risk domain, as physicians must take into account key areas of practice that differentiate it from other specialties. Accurate tumor staging, flow sheets, the need for multidisciplinary workflow documentation, integration of laboratory and imaging reporting, and dealing with chemotherapy ordering and toxicities are some of these unique demands [10]. Particular to the practice of oncology is the use of chemotherapeutic drugs, which have a lower therapeutic index than drugs used in other specialties, and for which a small dose miscalculation, or the misplacement of a decimal point in a dose value, can be fatal. The overarching goal of the present study was to assess the effects of interruptions on all tasks performed by oncologists during patient assessment and medication ordering work practices.

The few studies that have reported on the effects of interruptions during medication ordering have suggested interventions such as implementation of quiet work spaces where prescribers can order, review, and activate chemotherapy orders without interruption [4] and technological improvements such as computerized physician order entry systems [11]. These studies, however, provide little information on the specific nature and frequency of interruptions that occur throughout stages of the ordering process. Thus, despite the public health importance of the aforementioned studies, the patient safety impacts of interruptions on medication ordering errors are not well understood. There is a need to understand the nature and frequency of interruptions that occur at different stages of the medication ordering process, to (a) better inform the design of interruption mitigation strategies, and (b) guide healthcare managers/ executives on how to improve patient safety. Furthermore, investigations should not only focus on reducing interruptions, but must also identify circumstances when interruptions are a necessary form of communication. Strategies could then be developed to increase physicians' resiliency to interruptions. Thus, an important consideration is to assess how people react or cope with interruptions.

Certain coping strategies meant to deal with interruptions are more error-prone than others. Liu et al. [12] proposed the following 4 categories to describe mechanisms people can use to cope with interruptions: Engage (i.e., immediately attend to 
distraction), Multitask (i.e., attend to distraction while concurrently continuing to work on the task), Defer (i.e., acknowledge the request but continue primary task prior to addressing request), and Block (i.e., immediately indicate that they cannot deal with the distracting task and continue primary task). Liu et al. [12] suggest that engaging in interruptions or attempting to multitask may result in more errors than 'safer' coping mechanisms such as deferring or blocking interruptions. The results of Liu et al.'s study found that engaging and multitasking behaviours require clinicians to divert large attentional resources towards the interruption, and are therefore less safe mechanisms of coping with interruptions. On the other hand, deferring or blocking behaviours do not require drastic shifts in one's attention, and are consequently considered to be safer coping mechanisms.

The goals of the present study were (1) to understand the nature and frequency of interruptions during oncologists' patient assessments and ordering practices, and (2) to investigate oncologists' coping mechanisms when faced with an interruption.

\section{METHOD}

\subsection{Participants}

Three observers shadowed 7 oncologists for approximately 3 hours each, over a period of 2 months. A total of 38 shadowing sessions were conducted, with some oncologists being shadowed on several occasions. Shadowing sessions were conducted at different times throughout the workday (i.e., between 9 AM and 5 PM). Oncologists were informed of the general aims of the study through email notifications, and were invited to voluntarily participate after they consented to be shadowed. Ethics approval for this phase was obtained from the hospital's Research Ethics Board (Reference \#H2011:140).

\subsection{Location}

A cancer treatment teaching hospital in Canada, which administers outpatient intravenous (IV) chemotherapy to approximately 22,000 patients a year, was selected as the study location. This venue was selected because it is a setting in which high volumes of high-alert medications (in particular chemotherapy) are administered. Clinicians in this environment work in teams consisting of an oncologist, a clerk, and a nurse, and at times they also work with a clinic pharmacist.

\subsection{Procedure}

Trained observers who were familiar with oncologists' patient assessment and ordering processes gathered shadowing data. Each observer followed a single oncologist around the unit, while maintaining an appropriate distance and remaining as unobtrusive as possible to avoid influencing the oncologists' behaviour during the session. Once shadowing commenced, all tasks, interactions, and interruptions performed or experienced by the oncologist were recorded using a data collection tool. In addition, oncologists' methods of dealing with interruptions (i.e., coping mechanisms) were also measured according to a scheme proposed by Liu et al. [12]. 
In advance of the data collection phase, observers conducted pilot observations to practice using the tool and to collect inter-rater reliability data across observers. Specifically, 2 observers independently observed the same oncologist, and agreement between data elements (i.e., task, source and reason of interruption, time, and coping mechanism) was subsequently assessed through comparison. An inter-rater reliability analysis using the Kappa statistic was performed. If consistency was not achieved, discrepancies between transcripts were discussed until consensus was reached. Paired observations resumed until an inter-rater reliability level of substantial agreement (i.e., Kappa > 0.7) was reached amongst all observers.

All tasks were categorized in terms of their potential safety impact (i.e., low, medium, high). Level of safety impact was based on the predicted probability of an adverse event occurring during the conduct of the task. To determine safety impact, 3 raters individually categorized each task into 3 levels of potential safety impact. Discrepancies among raters were resolved by discussing the tasks and agreeing on a common category.

Reasons for interruption were determined during the pilot testing. After completion of the pilot testing, observers drafted a list of categories for the interruptions they observed. Observers interviewed the pilot participants to confirm what they observed. If a reason for interruption did not fall within the categorization developed in the pilot, observers noted the reason as "other".

For the purposes of observation, an interruption was defined as any externally initiated event that caused the oncologists' attention to be shifted from a primary task $[13,14]$. Depending on the nature of the interruption, the oncologist may or may not have paused primary task activity to attend to the external event. Therefore, this definition encompassed both interruptions and distractions. For instance, the oncologist may pause a medication verification task to attend to a telephone call (an interruption), or notice the telephone call but not attend to it (a distraction).

\subsection{Data Collection Tool}

All shadowing observations were electronically recorded on a Fujitsu Lifebook U810 microtablet PC. This computer was selected as it was highly portable, and allowed rapid data input through the use of a stylus. The software used to document oncologist tasks was the Remote Analysis of Team Environment (RATE) program [15]. This software was developed in the University of Virginia to track communications within a team setting, and allows users to score and annotate communication events in real time. This software was chosen as it allows for fast-paced events to be recorded and time-stamped rapidly. Observers conducted pre-shadowing observations to develop and refine a list of metrics tailored to the chemotherapy ordering environment. These metrics, which were then pre-programmed into RATE, included initiators of interactions/interruptions (e.g., oncologists, pharmacists, nurses, patients), physical locations (e.g., computer station, patient assessment room), tasks (e.g., physical exam, documentation), and coping mechanism used (i.e., engage/multitask vs. defer/block). The software also allowed tasks to be recorded within another pre-existing task (i.e., multitasking). For instance, an oncologist could be editing a medication order from 10:06:00 to 10:07:00, while also engaging in an interruption from 10:06:30 to 10:06:45. 


\subsection{Analysis}

All data collected during this experiment was analyzed using Microsoft Excel and the Statistical Package for the Social Sciences (SPSS, v18.0). An alpha level of .05 was used for all statistical tests. The main analyses included a comparison of percentage of interruptions for each task between all task types (i.e., [number of times a certain task is interrupted] / [total number of times this task is performed]), percent usage of coping mechanisms (i.e., [number of times a certain coping mechanism is used when performing a given task]/[total number times this task is interrupted), as well as a comparison of oncologists' average primary task completion time (i.e., time spent on primary task) between the conditions in which they were interrupted during their task performance and conditions in which they were uninterrupted. The percentage of interruptions for each task was analyzed in a 6 (task: travel vs. physical exam vs. documentation review vs. communication vs. documentation editing vs. dictation) oneway repeated measures ANOVA. The percentage use of coping mechanism was analyzed in a 6 (task: travel vs. physical exam vs. documentation review vs. communication vs. documentation editing vs. dictation) $\times 2$ (coping mechanism: engage/multitask vs. defer/block) repeated measures ANOVA. Finally, the average primary task completion time was analyzed in a 6 (task: travel vs. physical exam vs. documentation review vs. communication vs. documentation editing vs. dictation) $\times 2$ (status: non-interrupted vs. interrupted) repeated measures ANOVA. Pairwise comparisons were made using Bonferroni correction. Measures of effect size for ANOVAs were conducted using eta squared $\left(\eta^{2}\right)$ which represents the proportion of variance in the interruption rate, primary task completion time, and coping mechanism use (dependent variables) that is attributable to each effect (independent variables).

In studying the impact of interruptions on the time required for completion of primary task, one must consider the possibility that the longer it takes to complete a primary task, the greater the chance of that task being interrupted [16]. Therefore, "Time on Primary Task" for interrupted primary tasks may be longer than "Time on Primary Task" for uninterrupted primary tasks because interrupted primary tasks are inherently longer tasks, not because they are interrupted. When accounting for this latter possibility, Westbrook et al. [16] found that task completion times were shorter for interrupted tasks than for uninterrupted tasks. They hypothesize various reasons for this result including that clinicians, once interrupted, return to their primary task but compensate for the delay by hastening task completion.

In the present study, the total task time data are divided into (1) total time spent on performing primary task, and (2) total time spent on performing interrupted tasks. At times, however, there is an overlap between the "Time on Primary Task" and the "Time on Interruptions". That is, part or all of the "Time on Interruptions" can be embedded within the "Time on Primary Task". We have calculated the exact time spent on the interruptions ("Time on Interruptions") and the exact time spent on the primary task ("Time on Primary Task"), to assess whether the actual act of interrupting a primary task causes the task time to be lengthened. While the Time on Primary Task calculation includes all overlap with the Time on Interruptions regardless of the coping mechanism used, the Adjusted Time on Primary Task calculation subtracts overlap with the Time 
on Interruptions for certain coping mechanisms used. Specifically, different coping mechanisms are considered as follows:

- Blocked interruptions: "Adjusted Time on Primary Task" remains the same as the "Time on Primary Task" because the interruptions were blocked (i.e., no time spent dealing with interruptions; [Time on Interruptions] $=0$ ).

- Deferred interruptions: "Adjusted Time on Primary Task" is calculated by subtracting any overlap time between time spent on deferring the interruptions (e.g., asking interrupter to come back later) and Time on Primary Task from the "Time on Primary Task".

- Multitasked: "Adjusted Time on Primary Task" remains the same as the "Time on Primary Task" because the time spent on interruption is concurrent with the time spent on primary task when multitasking.

- Engaged in interruptions: "Adjusted Time on Primary Task" is calculated by subtracting any overlap between time spent engaging in interruptions and Time on Primary Task from the "Time on Primary Task", given that clinicians temporarily abandoned the primary task to address the interruption task when engaging in the interruptions. The resumption lag (i.e., time required to revert to primary task after completing interrupted task) is included in the time spent on interruptions.

The average primary task completion time for Adjusted Time on Primary Task was analyzed in a 6 (task: travel vs. physical exam vs. documentation review vs. communication vs. documentation editing vs. dictation) $\times 2$ (status: non-interrupted vs. interrupted) repeated measures ANOVA. Furthermore, to assess whether Adjusted Time on Primary Task varied with the coping mechanism used when confronted with the interruptions, we also conducted 4 separate 1-way (status: non-interrupted vs. interrupted) repeated measures ANOVAs for each of the coping mechanisms used (i.e., block, defer, multitask, engage).

\section{RESULTS}

\subsection{General Observations}

Oncologists were responsible for assessing patients and ordering medications. They documented multiple orders for complicated regimens, involving tasks such as calculations (e.g., body surface area, creatinine clearance, area under the curve), consideration and integration of patient history and patient age, previous drug exposure, toxicities, and specific patient characteristics (e.g., allergies). Oncologists usually performed patient assessments on their own in private rooms dedicated to these assessments. When ordering medications, oncologists depended on communication with their teams usually consisting of a nurse, a clerk and at times a clinic pharmacist. Information exchanges among team members were numerous but typically brief. Ordering of medications took place in a room where oncologists sat side-by-side with their team members. There were typically four teams working in a medication-ordering room. Oncologists ordered medications using a computerized physician order entry system as well as paper-based forms. 
There were a total of 76 hours of observations, with 8444 interruptions observed. A total of 7 sources of interruptions (i.e., patient, nurse, other oncologist, patient's relative/guest, clinic pharmacist, triage pharmacist, and clerk) and 7 reasons for interruptions [i.e., communications related to (1) assessments, (2) bloodwork, (3) patient history, (4) medication orders, (5) documentation, (6) other work-related issues, and (7) personal] were observed. The following 6 tasks were observed: travel, physical exam, documentation review (electronic and paper formats), communication (i.e., discussions, phone calls, emails, pages, faxes), documentation editing (electronic and paper formats), dictations. The unit evaluated in the present study used a dual paper/electronic medication order system, requiring physicians to verify orders against both system types. All tasks were subsequently categorized in terms of their potential safety impact (i.e., low, medium, high). Table 1 provides a description of tasks and categorizations.

\subsection{Overall Interruption Data}

Oncologists were frequently interrupted (621 interruptions observed) during their ordering process, up to 19 interruptions per hour (mean: 8 interruptions/hour, range: 1 to 19$)$. On average, oncologists spent $17 \%$ of their total work time) on interruptions.

\subsection{Frequency of Interruptions for Each Task}

Figure 1 displays the percentage of interruptions per task occurrence (i.e., [Number of times a certain task is interrupted] / [Total number of times this task is performed]). The percentage of interruption varied significantly across tasks $[\mathrm{F}(5,135)=4$, $\mathrm{p}<.003$ $\left.\left(\eta^{2}=0.13\right)\right]$. Specifically, the frequency of interruption was significantly higher for physical exam (mean: 20\%), documentation review (mean: 18\%), and documentation editing (mean: 18\%; all pairwise comparisons $\mathrm{p}<.01$ ) compared to travel (mean: $7 \%$ )

Table 1. Task categorization and descriptions

\begin{tabular}{|c|c|c|}
\hline Potential safety impact & Task & Task description \\
\hline \multirow[t]{2}{*}{ Low } & Travelling & $\begin{array}{l}\text { Walking to and from different locations } \\
\text { within the unit }\end{array}$ \\
\hline & Physical examination & $\begin{array}{l}\text { Conducting a physical assessment of the } \\
\text { patient }\end{array}$ \\
\hline \multirow[t]{2}{*}{ Medium } & Documentation review & $\begin{array}{l}\text { Reviewing either electronic or paper } \\
\text { document transcribed from dictation, but } \\
\text { NOT editing }\end{array}$ \\
\hline & Communication & $\begin{array}{l}\text { Answering questions, discussing, faxing, } \\
\text { emailing }\end{array}$ \\
\hline \multirow[t]{2}{*}{ High } & Documentation editing & $\begin{array}{l}\text { Adding, changing, or deleting words in } \\
\text { an electronic or paper document }\end{array}$ \\
\hline & Dictation & $\begin{array}{l}\text { Verbally recording the assessment and } \\
\text { patient visit event into a phone system }\end{array}$ \\
\hline
\end{tabular}




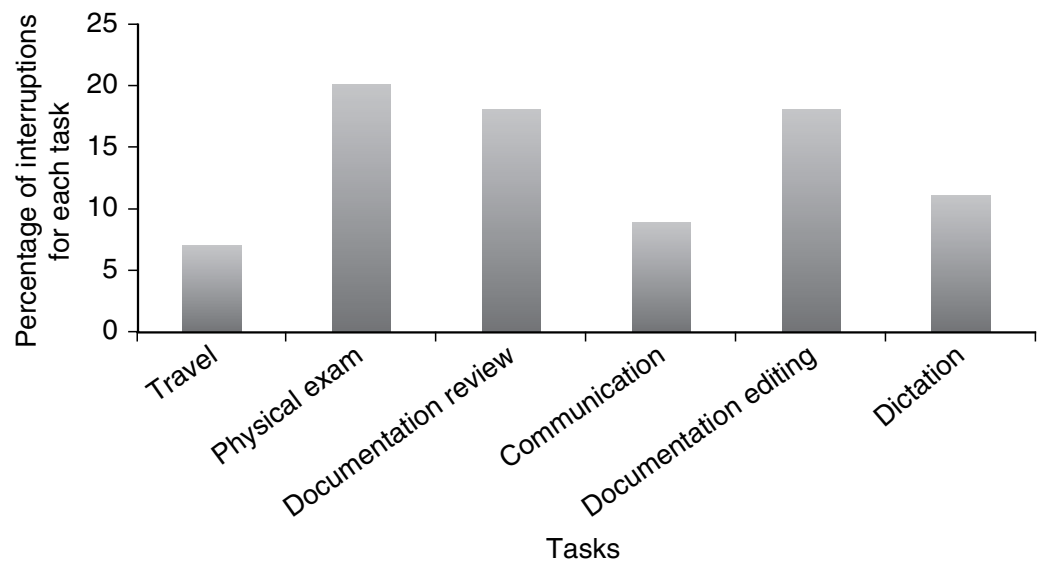

Figure 1. Percentage of interruptions for each task $(\mathrm{N}=8444$ interruptions observed).

and communication (mean: 9\%). Dictation (mean: 11\%) did not differ significantly from any of the other tasks.

\subsection{Sources of Interruptions During High-Safety Impact Tasks}

Documentation editing and dictation are categorized as safety-critical tasks. As displayed in Figure 2, the main sources of interruptions during safety-critical tasks were nurses (38\%), followed by other oncologists (20\%), and patients (17\%). Other sources of interruption were clinic pharmacists $(16 \%)$, clerks $(6 \%)$, phone calls $(2 \%)$, and patients' relative/guests $(1 \%)$.

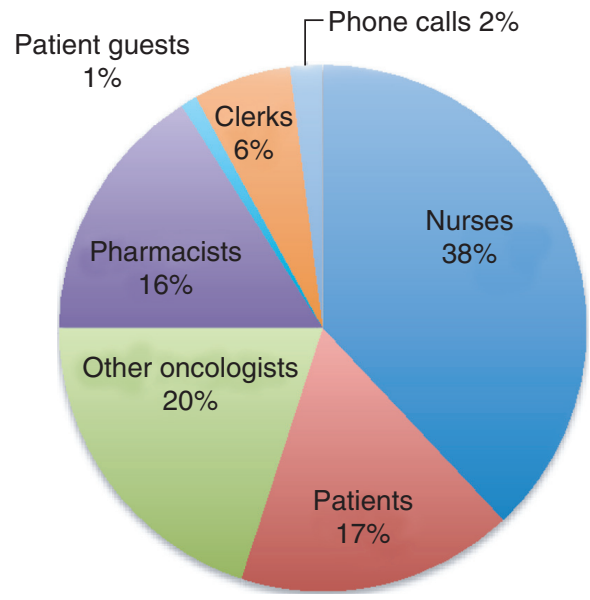

Figure 2. Sources of interruptions for safety critical tasks $(\mathrm{N}=124$ interruptions observed). 


\subsection{Reasons for Interruption Across All Tasks}

Table 2 presents a list of the primary interruption sources and reasons for interruption for each task. Interruptions occurred mainly for communicative reasons. The following provides a description of the different categories of reasons for interruptions:

1. Medication orders: communication event regarding a patient-specific order including drug type, dosage, or protocol.

2. Patient history: communication event concerning patient history (e.g., prior medical conditions, drug allergies).

3. Patient assessment: communication event between oncologist and a patient, patient's relative/guest, or nurse, related to the oncologist's assessment of the patient in the observation room.

4. Bloodwork: communication event regarding a patient's bloodwork status (e.g., nurse asking oncologist whether bloodwork results have been received from lab).

5. Other related work: communication event that is clearly work-related but does not fall into any of the main identified categories above.

6. Personal: communication unrelated to work (e.g., nurse asking oncologist about plans for the weekend).

Table 2. Levels of potential safety impact, tasks, interruption sources, and interruption reasons

\begin{tabular}{|c|c|c|c|}
\hline $\begin{array}{l}\text { Potential } \\
\text { safety } \\
\text { impact }\end{array}$ & Task & $\begin{array}{l}\text { Primary source of } \\
\text { interruption }\end{array}$ & Primary reason for interruption \\
\hline Low & Travel & Nurses & $\begin{array}{l}\text { Patient assessment and patient history } \\
\text { questions }\end{array}$ \\
\hline Low & Physical exam & Patients & $\begin{array}{l}\text { Patient assessment and medication order } \\
\text { questions }\end{array}$ \\
\hline \multirow[t]{3}{*}{ Medium } & $\begin{array}{l}\text { Documentation } \\
\text { review: }\end{array}$ & & \\
\hline & Electronic & $\begin{array}{l}\text { Fellow oncologists } \\
\& \text { nurses }\end{array}$ & Patient history questions \\
\hline & Paper & Patients & Patient assessment questions \\
\hline Medium & Communication & $\begin{array}{l}\text { Patients and Patients' } \\
\text { relatives/guests }\end{array}$ & Patient assessment questions \\
\hline \multirow[t]{3}{*}{ High } & $\begin{array}{l}\text { Documentation } \\
\text { editing: }\end{array}$ & & \\
\hline & Electronic & Nurses & $\begin{array}{l}\text { Patient history and medication order } \\
\text { questions }\end{array}$ \\
\hline & Paper & Patients & $\begin{array}{l}\text { Patient assessment and medication order } \\
\text { questions }\end{array}$ \\
\hline
\end{tabular}




\subsection{Reasons for Interruption During High-Safety Impact Tasks}

The reasons for interruption during safety-critical tasks were as follows: questions pertaining to medication orders $(33 \%, \mathrm{n}=42)$, questions pertaining to patient history $(31 \%, \mathrm{n}=39)$, other work-related questions $(18 \%, \mathrm{n}=23)$, questions pertaining to patient assessment $(11 \%, \mathrm{n}=14)$, personal discussions $(5 \%, \mathrm{n}=6)$, and questions pertaining to blood work $(2 \%, \mathrm{n}=2)$. The main source and reason for interruption varied with the type of task performed when the interruption occurred.

\subsection{Coping Mechanisms}

The primary purpose of the coping mechanism analysis was to assess whether oncologists used safer coping mechanisms (i.e., defer and block) compared to less safe coping mechanisms (i.e., engage and multitask). Therefore, the 4 coping codes were further condensed into Engage/Multitask and Defer/Block. As shown in Figure 3, when interrupted during all tasks, oncologists engaged and multitasked significantly more often than deferring and blocking (see Table 3 for ANOVA results). Therefore, the present analysis reveals that oncologists adopted less safe mechanisms to cope with interruptions.

\subsection{Average Time on Primary Task with or without Interruptions}

Average Time on Primary Task varied significantly with task type (i.e., travel, physical exam, documentation review, communication, documentation editing, dictation) and with or without interruption. As displayed in Figure 4, the average Time on Primary Task for all tasks, with the exception of communication, were significantly longer if interrupted than not interrupted (see Table 4 for ANOVA results).

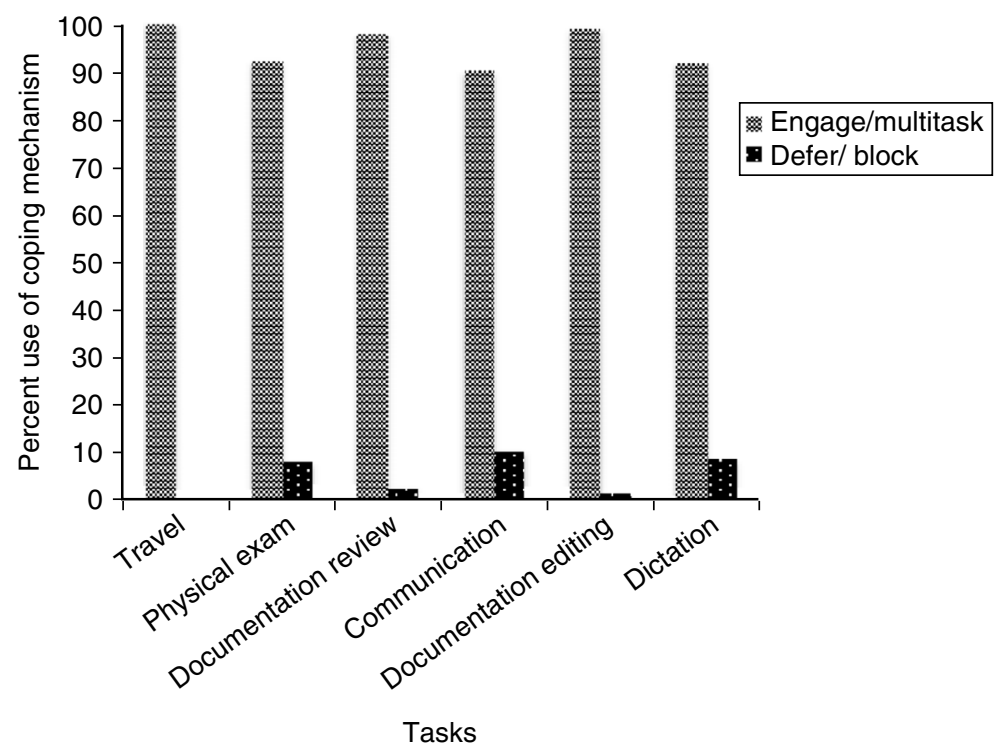

Figure 3. Percent use of coping mechanisms for different tasks $(\mathrm{N}=988)$. 
Table 3. ANOVA results for coping mechanism analysis

\begin{tabular}{lccc}
\hline Factor & Test statistic & Significance & Variance explained \\
\hline Tasks & $\mathrm{F}(5,95)=5$ & $(\mathrm{p}<0.002)^{*}$ & $\eta^{2}=0.20$ \\
Coping mechanism & $\mathrm{F}(1,19)=9$ & $(\mathrm{p}<0.01)^{*}$ & $\eta^{2}=0.32$ \\
Tasks $\times$ Coping mechanism & $\mathrm{F}(5,95)=3$ & $(\mathrm{p}>0.05)$ & $\eta^{2}=0.02$ \\
\hline
\end{tabular}

* Significant at the 0.05 level

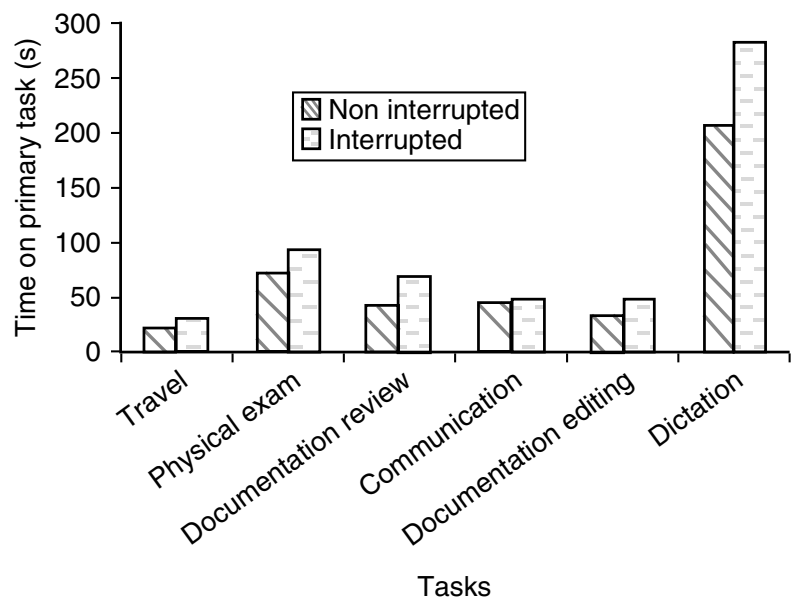

Figure 4. Average primary task completion time for different tasks with and without interruptions.

Table 4. ANOVA results for average time on primary task with or without interruption

\begin{tabular}{lccc}
\hline Factor & Test statistic & Significance & Variance explained \\
\hline With or without interruption & $\mathrm{F}(1,94)=238$ & $(\mathrm{p}<.001)^{*}$ & $\eta^{2}=0.72$ \\
Task & $\mathrm{F}(5,470)=1258$ & $(\mathrm{p}<.001)^{*}$ & $\eta^{2}=0.93$ \\
With or without interruption $\times$ Task & $\mathrm{F}(5,470)=32$ & $(\mathrm{p}<.001)^{*}$ & $\eta^{2}=0.25$ \\
\hline
\end{tabular}

* Significant at the 0.05 level

\subsection{Adjusted Average Time on Primary Task with or without Interruption}

After adjusting the Time on Primary Task, we did not find a significant difference in the Adjusted Times on Primary Task with and without interruption (see Table 5 for ANOVA results). Adjusted Time on Primary Task for completing "dictation" was significantly longer compared to all other tasks. 
Table 5. ANOVA results for adjusted average time on primary task with or without interruption

\begin{tabular}{lccc}
\hline Factor & Test statistic & Significance & Variance explained \\
\hline With or without interruption & $\mathrm{F}(1,94)=20$ & $(\mathrm{p}>.05)$ & $\eta^{2}=0.002$ \\
Task & $\mathrm{F}(5,470)=107$ & $(\mathrm{p}<.05)^{*}$ & $\eta^{2}=0.78$ \\
With or without interruption $\times$ Task & $\mathrm{F}(5,470)=3$ & $(\mathrm{p}>.05)$ & $\eta^{2}=0.19$ \\
\hline
\end{tabular}

* Significant at the 0.05 level

Table 6. ANOVA results for adjusted average times on primary task as a function of interruption and coping mechanism

\begin{tabular}{lccc}
\hline Coping mechanism & Test statistic & Significance & Variance explained \\
\hline Block & $\mathrm{F}(1,10)=0.02$ & $(\mathrm{p}>.05)$ & $\eta^{2}=0.002$ \\
Defer & $\mathrm{F}(1,14)=0.95$ & $(\mathrm{p}>.05)$ & $\eta^{2}=0.06$ \\
Multitask & $\mathrm{F}(1,301)=44$ & $(\mathrm{p}<.001)^{*}$ & $\eta^{2}=0.1$ \\
Engage & $\mathrm{F}(1,193)=15$ & $(\mathrm{p}<.001)^{*}$ & $\eta^{2}=0.1$ \\
\hline
\end{tabular}

* Significant at the 0.05 level

However, we found that the effect of interruption status on Adjusted Times on Primary Task varied with coping mechanism adopted. Specifically, results in Table 6 revealed no significant difference in Adjusted Times on Primary Task for the "block" and "defer" mechanisms compared to non-interrupted conditions. Significant differences, however, were found for the "multitask" and "engage" mechanisms. Specifically, Adjusted Times on Primary Task were significantly longer when oncologists "multitasked" (mean = $63.6 \mathrm{~s}$ ) compared to uninterrupted conditions $($ mean $=39.5 \mathrm{~s})$. Conversely, Adjusted Times on Primary Task were significantly shorter when oncologists "engaged" (mean $=26.4 \mathrm{~s})$ compared to uninterrupted condition $($ mean $=38.9 \mathrm{~s})$.

\section{DISCUSSION}

A direct observational study was conducted to qualitatively and quantitatively describe the state of interruptions for medical oncologists in a Canadian cancer treatment facility. The present work supports conclusions from published studies that interruptions to clinicians are ubiquitous in various healthcare settings [17-19].

A large number of interruptions (see Figure 1) occurred during tasks categorized as safety critical (i.e., documentation editing and dictation). The high rate of errors during documentation editing tasks is of particular interest given that previous studies [20,21] have shown a disruptive effect of interruptions on complex cognitive operations in computer-based editing tasks. For example, Magrabi et al. [21] found that the disruptive effects of interruptions were more pronounced during electronic prescribing tasks 
requiring editing of medications compared to those requiring entering information status because the later required less information cues to complete the task. Therefore, although we did not assess the effects of interruptions on error rates, results from these previous studies suggest that the "documentation editing" task in the current study may be especially prone to disruptive effects of interruptions.

Given the higher potential safety impact associated with documentation editing, interventions should be implemented to mitigate risks associated with interruptions during this task. Pape et al. [22] found that the use of Do Not Disturb signage significantly decreased interruptions to nurses during medication administration tasks. However, they also acknowledged that habituation effects may limit the effectiveness of signage. Other researchers [23] have also found that interventions aimed at increasing awareness of interruptions through education of nurses and patients were successful in decreasing the rate of interruption. Thus, potential interventions to reduce the interruptions during documentation editing could include incorporating dedicating physical spaces, with targeted "Do Not Disturb" signage in clinical settings for documentation editing.

Interruptions have also been found to disrupt cognitive processes, increase stress, and cause confusion or omission of tasks [12, 19, 24]. Furthermore, Westbrook et al. [16] showed that occurrence and frequency of interruptions was significantly associated with the incidence of procedural failures and clinical errors, and that error severity increased with interruption frequency. Thus, there is a high risk that interruptions can lead to medication errors with the potential to cause serious harm to patients.

Nurses, patients, and other oncologists were found to be the most frequent sources of interruption, with interruptions occurring due to communication-related reasons (e.g., asking questions related to patient assessments, patient history, and medication orders). Although we did not set out to quantify the number of times that face-to-face communication events seeking information (e.g., nurse asking oncologist patienthistory-related questions) that could have been accessed from other sources (e.g., nurse looking up patient history information in patient chart), oncologists did informally mention to observers that information requested by their colleagues could at times be found elsewhere (e.g., patient chart, electronic order). This is consistent with past researchers [25] who have reported that physician teams were subject to high levels of interruptions and consequently bore a higher cognitive load than necessary. That is, many questions that could be answered by accessing information sources were instead raised to physicians. Researchers [26] suggest that such interruptions impose cognitive load on clinical staff and can thus impact memory and lead to clinical errors. Similarly, researchers [27] found that communication behaviour in healthcare settings might result in an interruptive work environment and contribute to inefficiency. Thus, the results of the present study support other researchers' [25-27] conclusions for the need for communication training in healthcare workspaces. Further research is needed to investigate how to best manage the apparent bias towards interruptive communication channels such as interpersonal exchanges (e.g., face-to-face conversations) rather than less interruptive channels such as computer-mediated communication (e.g., electronic message boards). 
Interruption must not necessarily be viewed negatively. Grundgeiger and Sanderson [24] suggest that interruptive communication may actually provide a means of transmitting urgent and valuable information to the person being interrupted. The chemotherapy ordering process often requires oncologists to interact with other health professionals such as fellows, residents, pharmacists, other oncologists, nurses, and clerks, to share information. Therefore, practitioners engaged in managing and contributing to chemotherapy ordering must maintain a shared assessment of the situation.

The need for shared situation assessments is common in other industries (e.g., aviation, chemical and nuclear process control) where practice involves the management and control of dynamic systems. These domains are similar to healthcare in that they often require interpretation of a situation before all the data are available. For example, oncologists must often place a medication order while still waiting on patients' blood test results. Understanding how to support a team member's situation assessments is an important consideration when assessing interruption mitigation strategies. That is, solutions that aim to reduce interruptions caused by communication must incorporate other compensatory ways of supporting team members' common situation assessment. The results of the present study provide a first step in identifying some of the general categories (e.g., questions pertaining to patient history or bloodwork results) of information that team members, who are engaged in the chemotherapy ordering process, need to exchange. This information is vital to the design of effective interventions to improve safe chemotherapy ordering.

When an interruption is in relation to the actual task, one might also consider it as part of task at hand (i.e., part of the conversation within the team) instead of considering it an interruption. However, even though communication is needed between team members, there are times when it is inappropriate (i.e., unsafe) to interrupt. As such, interventions are needed to raise awareness of an opportune time versus a risky time to communicate and/or interrupt even if the communication/interruption is related to the task at hand. The ultimate goal is not to eliminate all interruptions but rather to reduce unnecessary interruptions. For example, interruptions could be minimized during safety critical tasks. Furthermore, redundancies could be built into systems (i.e., systems could be built to recover or adapt to change while preserving essential properties) to help mitigate risks associated with interruptions during safety critical tasks. It is important, for example, that multiple mechanisms (e.g., technologies) be employed to communicate critical information. This allows a shift among mechanisms depending on the demands of the system, or the potential malfunction of any particular type of mechanism.

Nurses' mechanisms of coping with interruptions were coded as Engage, Multitask, Defer, and Block, according to the scheme proposed by Liu et al. [12]. The results of Liu et al.'s experiment suggested that engaging and multitasking behaviours require clinicians to divert large attentional resources towards the interruptions, and are consequently unsafe mechanisms to cope with interruptions. Conversely, deferring or blocking behaviours do not require drastic shifts in nurses' attention, and are thus considered to be safe coping mechanisms. Results of the present study revealed that 
oncologists used unsafe coping mechanisms (engaged or multitasked) when interrupted, significantly more often than safe coping mechanisms (deferred or blocked).

The present results revealed that depending on the coping mechanism used, Adjusted Time on Primary Task may or may not be impacted by the interruption. Our findings show that Adjusted Times on Primary Task are longer for interrupted tasks than for tasks without interruptions when "multitasking" is the coping mechanism. Conversely, our results show that Adjusted Times on Primary Task are shorter for interrupted tasks than for tasks with no interruptions when "engaging" is the coping mechanism adopted. This latter result is consistent with Westbrook et al.'s [16] findings that clinicians reduce the time they spend on tasks if they experience interruptions. No significant differences were found in Adjusted Times on Primary Task for interrupted tasks compared to uninterrupted tasks when "deferring" and "blocking" coping mechanisms were adopted. This finding of the "deferring and blocking" strategies may be limited, however, given the potentially lower statistical power for these analyses since there were fewer occurrences of these strategies compared to "multitasking" and "engaging". Nonetheless, these findings reveal that the relationship between interruptions and primary task completion times may vary depending on the coping mechanism. Our findings suggest that the conflicting findings across researchers about the effects of interruptions on task completion times might be due to failure to consider coping behaviour used.

It is also interesting to consider our findings in light of Liu et al.'s [12] suggestion that deferring and blocking behaviours are safer than engaging and multitasking behaviours because deferring and blocking do not require drastic shifts in attention, whereas engaging and multitasking do. The present findings support Liu et al.'s [12] notion that engaging and multitasking behaviours have a larger impact on task performance than deferring and blocking. However, the fact that engaging and multitasking behaviours lead to opposite effects on task completion time suggest that there may be other explanations besides drastic shifts in attention for these results. Our study does not provide an explanation, but several explanations are possible. For example, "multitasking" might increase task completion time because it requires completion of two or more tasks at once, whereas "engaging" might shorten task completion time because people compensate for the time spent on the interruption by rushing task completion. Further research is needed to provide additional insight into the effects of coping behaviour on task completion time. Nonetheless, our study raises some valuable analysis considerations when examining effects of interruptions on task completion time. Failing to consider coping mechanism used when faced with an interruption could lead to incorrect conclusions.

In sum, completely eliminating sources of interruption is neither feasible nor recommended. Instead, efforts should be made to minimize the impact of interruptions during safety-critical tasks and/or to increase oncologists' resiliency to interruptions. Thus, there is a need for interventions targeted at (1) helping oncologists to mitigate the harmful effects of interruptions during medication ordering tasks, and (2) encouraging oncologists to use safe coping mechanisms when confronted with interruptions.

There were limitations to this study. First, as with any observational study, there is a risk that the presence of observers might have influenced the activity patterns of the 
oncologists being followed. In other words, it is possible that oncologists may have modified their behavior from what it would have been without the knowledge that they were being observed, referred to as the Hawthorne Effect [28]. Although steps were taken to ensure that observers' presence was as unobtrusive as possible to the oncologists, it was impossible to guarantee that oncologists' behaviour remained unchanged as a result of being observed. However, any behaviour change resulting from the Hawthorne Effect is only likely to have increased vigilance of staff while being observed. Thus, observations discovered under these circumstances can reasonably be considered best-case outcomes. Second, this study was conducted in a single hospital. The risk of interruptions and their potential impact on medication ordering practices may vary across sites. Therefore, the study results may not be representative of cancer treatment facilities throughout Canada and other countries.

\section{CONCLUSION}

Oncologists working in cancer treatment facilities are frequently interrupted during safety-critical tasks. The present findings show that interruptions differentially impact task efficiency depending on the coping mechanism adopted. More importantly, however, interruptions can lead to errors with potentially catastrophic consequences. Unlike other industries, such as aviation, the effects of interruptions on medication errors have not yet been systematically studied in healthcare, especially at the ordering stage of the process. The present study provides an initial step to understanding the nature, frequency, causes, and effects of interruptions during chemotherapy ordering. Mitigating the effects of interruptions to reduce medical errors and increase efficiency in such an environment is of great importance to patient safety and healthcare quality.

\section{ACKNOWLEDGEMENTS}

The authors thank the study hospital staff, and Stacey Howchin and Nancy Ho for their help in the data collection.

\section{CONFLICT OF INTEREST}

The authors indicated no potential conflicts of interest.

\section{REFERENCES}

[1] Kohn LT, Corrigan JM, Donaldson MS, eds. To Err is Human: Building a Safer Healthcare System. Washington, D.C.: Institute of Medicine. National Academy Press; 2000.

[2] Schwappach D.L.B. \& Wernli M. Medication errors in chemotherapy: incidence, types and involvement of patients in prevention. A review of the literature. European Journal of Cancer Care, 2010, 19:285-292.

[3] ISMP Canada. Fluorouracil incident root cause analysis, 2007. http://www.ismpcanada.org/ download/reports/FluorouracilIncidentMay2007.pdf. Accessed November, 2012.

[4] Womer RB, Tracy E, Soo-Hoo W, Bickert B, DiTaranto S, Barnsteiner JH. Multidisciplinary systems approach to chemotherapy safety: Rebuilding processes and holding the gains. J Clin Oncol, 2002, 20:4705-4712.

[5] Trbovich PL, Prakash V, Stewart J, Trip K, Savage P. Interruptions during the Delivery of High Risk Medications. Journal Of Nursing Administration, 2010, 40(5):211-218. 
[6] Hickam DH, Severance SS, Feldstein A, Ray L, Gorman P, Schuldheis S, Hersh WR, Pyle Krages K. The Effect of Health Care Working Conditions on Patient Safety. Agency for Healthcare Research and Quality. 2003;74.

[7] Summary of Information Submitted to MEDMARX in the Year 2001: A Human Factors Approach to Understanding Medication Errors. Rockville, MD: United States Pharmacopeia; December 2002.

[8] United States Pharmacopeia. USP Patient Safety CAPSLink September 2003. USP Patient Safety CAPSLink. http://us.vocuspr.com/Newsroom/ViewAttachment.aspx?SiteName=USPharm\&Entity= PRAsset\&AttachmentType $=F \&$ EntityID=73530\&AttachmentID=7e173c5e-8315-4f77-92c0ce061c17912e. Accessed November, 2012.

[9] Leape LL, Bates DW, Cullen DJ, Cooper J, Demonaco HJ, Gallivan T, Hallisey R, Ives J, Laird N, Laffel J. Systems Analysis of Adverse Drug Events, JAMA, 1995, 274(1):35-43.

[10] American Society of Clinical Oncology: The Oncologist's Field Guide to Selecting and Implementing an Electronic Health Record. Alexandria, VA, ASCO, 2008. http://www.asco.org/ASCOv2/Meetings/ Annual+Meeting/Past+Annual+Meetings/2008+Annual+Meeting/2008+ASCO+Daily+News/Monday, +June+2,+2008+Section+B/ASCO+Develops+Practical+Guide+for+Selecting,+Implementing+Elect ronic+Health+Records. Accessed November, 2012.

[11] Shulman LN, Miller RS, Ambinder EP, Yu PP, Cox JV. Principles of Safe Practice Using an Oncology EHR System for Chemotherapy Ordering, Preparation, and Administration, Part 1 of 2. J Oncol Pract., 2008, 4(4):203-206.

[12] Liu D, Grundgeiger T, Sanderson PM, Jenkins SA, Leane TA. Interruptions and blood transfusion checks: lessons from the simulated operating room. Anesthesia and analgesia, 2009,108(1):219-222.

[13] Ebright PR, Patterson ES, Chalko BA, Render ML. Understanding the complexity of registered nurse work in acute care settings. $J$ Nurs Adm., 2003, 33(12):630-638.

[14] Drews FA. The frequency and impact of task interruptions in the ICU. Proceedings of the 51st Annual Meeting of the Human Factors and Ergonomics Society, 2007:683-686.

[15] Osborne N, Shin T, Guo H, Gerlain S. Remote Analysis of Team Environments (RATE). University of Virginia. 2004; 2.0.0.0. http://www.sys.virginia.edu/hci/Reference/RATE\%20Manual.pdf . Accessed November 2012.

[16] Westbrook JI, Coiera ED, W.T.M., Brown BM, Kelk N, Paoloni R, Tran C. The impact of interruptions on clinical task completion. Qual Saf Health Care, 2010, 19:284-289.

[17] Alvarez G, Coiera E. Interruptive communication patterns in the intensive care unit ward round. Int $J$ Med Inf., 2005. 74(10):791-796.

[18] Chisholm CD, Dornfeld AM, Nelson DR, Cordell WH. Work interrupted: A comparison of workplace interruptions in emergency departments and primary care offices. Ann Emerg Med., 2001, 38:146-151.

[19] Parker J, Coiera E. Improving clinical communication: A view from psychology. JAMIA, 2000;7(5):453-461.

[20] Burmistrov I, Leonova A. Do interrupted users work faster or slower? the micro-analysis of a computerized text editing task. In: Jacko J, Stephanidis C, editors., eds. Human-computer interaction: theory and practice (Part I). Mahwah, New Jersey: Lawrence Erlbaum Associates, 2003:621-5.

[21] Magrabi F, Li SY W, Day R, Coiera E. Errors and electronic prescribing: a controlled laboratory study to examine task complexity and interruption effects. Journal of the American Medical Informatics Association, 2010, 17:575-583.

[22] Pape TM, Guerra DM, Muzquiz M. Innovative Approaches to Reducing Nurses' Distractions During Medication Administration. The Journal of Continuing Education in Nursing. 2005, 36(3):108-116.

[23] Relihan E, O'Brien V, O'Hara S, Silke B. The impact of a set of interventions to reduce interruptions and distractions to nurses during medication administration. Qual Saf Health Care. 2010, 19(5):e52.

[24] Grundgeiger T, Sanderson P. Interruptions in healthcare: Theoretical views. Int J Med Inf., 2009, 78(5):293-307.

[25] Coiera, W, Jayasuriya R, Hardy J, Bannan A, Thorpe M. Communication loads on clinicians in the Emergency Department. Medical Journal of Australia, 2002, 176(9):415-418. 
[26] Parker J, Coiera E. Improving clinical communications: A view from psychology. Journal of the American Medical Informatics Association, 2000, 7(5):453-461.

[27] Coiera E, Tombs V. Communication behaviours in a hospital setting: an observational study. British Medical Journal, 1998, 316:673-676.

[28] Parsons, HM. What happened at Hawthorne? Science, 1974, 183(4128):922-32. 


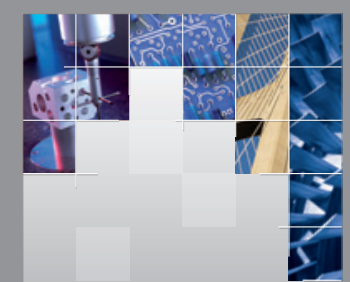

\section{Enfincering}
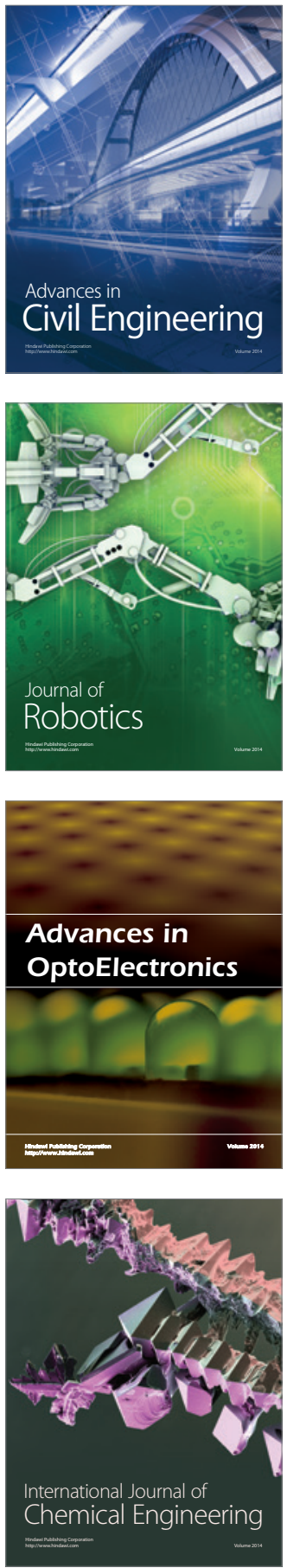

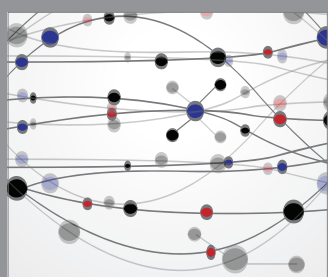

The Scientific World Journal

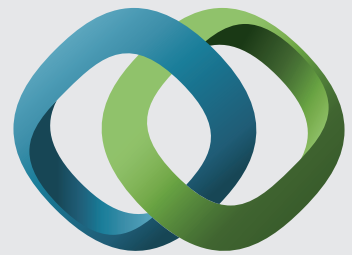

\section{Hindawi}

Submit your manuscripts at

http://www.hindawi.com
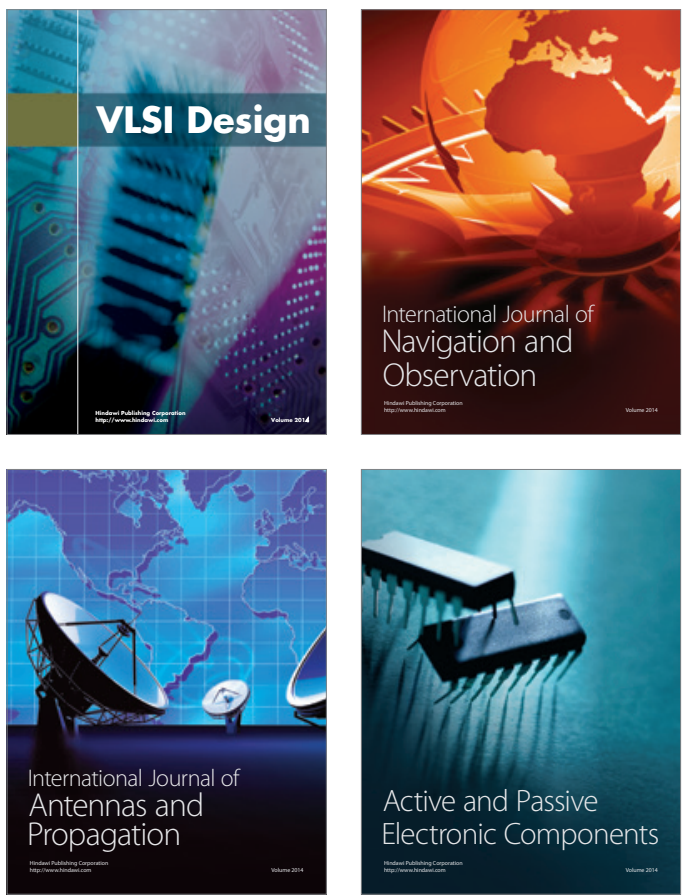
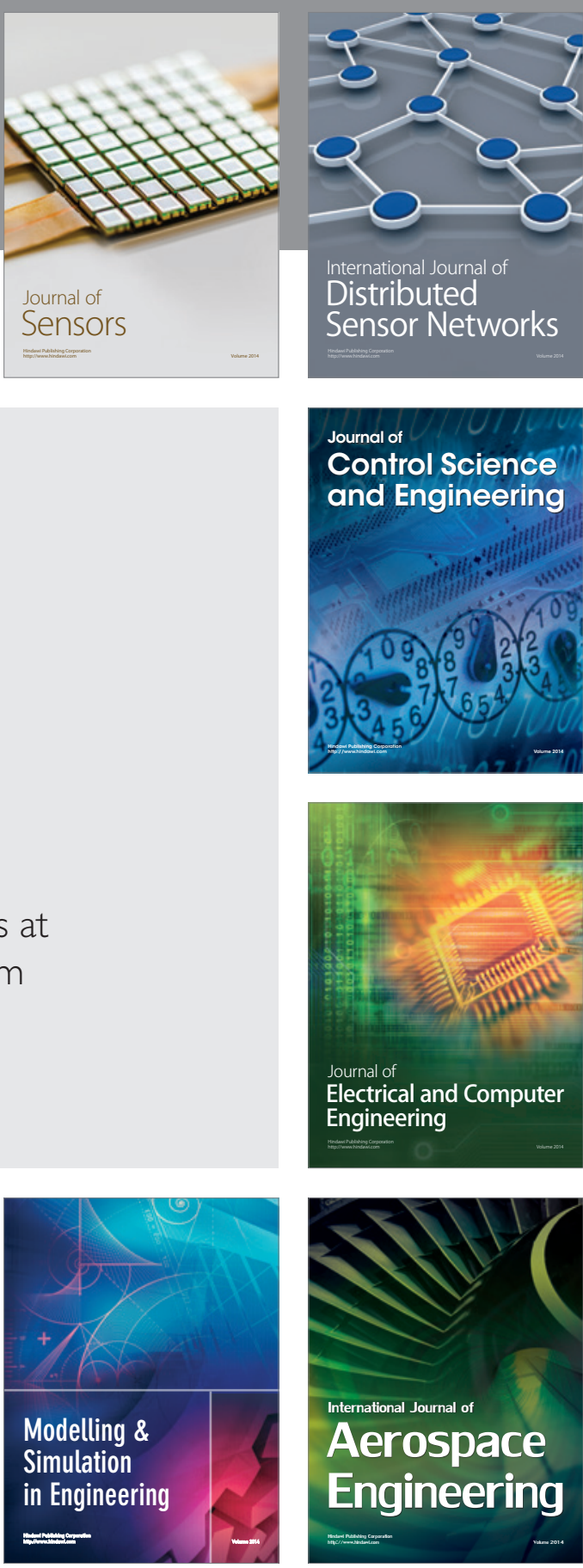

International Journal of

Distributed

Sensor Networks

Journal of

Control Science

and Engineering
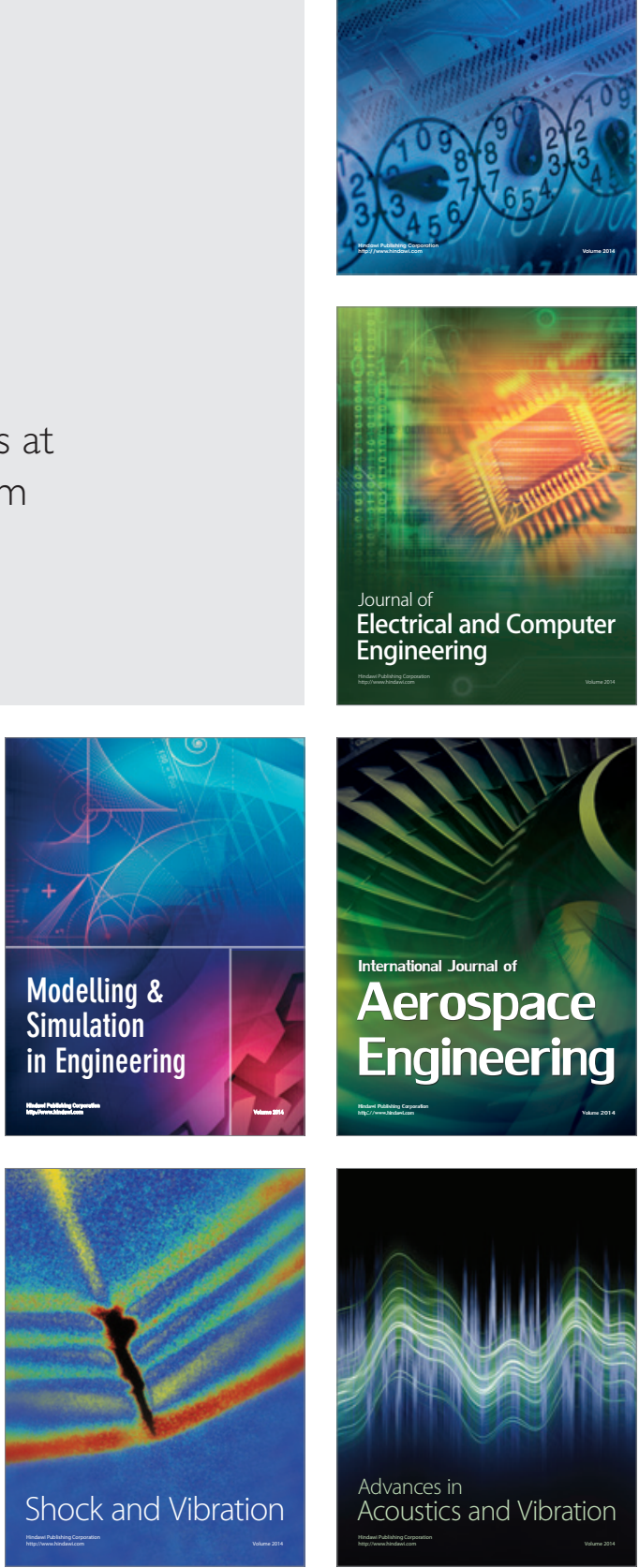